ULM-TP/97-10

October 1997

\title{
Billiard Systems in Three Dimensions: \\ The Boundary Integral Equation and the Trace Formula
}

\author{
Martin Sieber \\ Abteilung Theoretische Physik, Universität Ulm, D-89069 Ulm, Germany
}

\begin{abstract}
We derive semiclassical contributions of periodic orbits from a boundary integral equation for three-dimensional billiard systems. We use an iterative method that keeps track of the composition of the stability matrix and the Maslov index as an orbit is traversed. Results are given for isolated periodic orbits and rotationally invariant families of periodic orbits in axially symmetric billiard systems. A practical method for determining the stability matrix and the Maslov index is described.
\end{abstract}

PACS numbers:

03.65.Sq Semiclassical theories and applications.

05.45.+b Theory and models of chaotic systems. 


\section{Introduction}

Billiards are popular models for the study of dynamical systems and their quantum counterparts. They are simpler than general systems with a potential and exhibit a large spectrum of dynamical behaviour ranging from integrability to chaoticity. Until recently, most investigations have concentrated on two-dimensional billiards whose numerical treatment requires much less effort than higher-dimensional systems. But two-dimensional systems also have rather special properties like the division of the energy surface into separate regions by invariant tori.

During the last three years there have been several publications on three-dimensional billiard systems as models for chaotic systems [1, 2, 3, 4, 5, 6, 7, 8, 9, 10, 11, 12]. In other fields threedimensional billiard systems have served as models much longer, like in nuclear or clusters physics. An overview on these applications is given in [13]. In this context, Balian and Bloch investigated in detail semiclassical approximations for the level density in three-dimensional billiard systems 114, 15, 16]. Their results for the contributions of periodic orbits are expressed in terms of determinants of $2 n$-dimensional matrices, where $n$ is the number of reflection points of a trajectory. In contrast to this, the results of Gutzwiller for smooth three-dimensional systems are expressed in terms of the $4 \times 4$-stability matrix [17], and the relation between both results is not explicit.

For two dimensional billiard systems Harayama and Shudo derived semiclassical contributions of isolated periodic orbits in terms of the stability matrix starting from a boundary integral equation [18]. Their derivation involved the transformation of $n$-dimensional matrices, where again $n$ is the number of reflections of a trajectory. For higher dimensional systems this method would be very elaborate. In addition, the relation between the obtained index in the trace formula and the number of conjugate points is not direct [19].

We avoid this difficulty by applying an iterative method that follows the trajectory from reflection to reflection and keeps track of the composition of the stability matrix and the Maslov index as the trajectory is traversed. This method has proven to be convenient for the derivation of uniform approximations for diffractive orbits in two-dimensional billiard systems [20].

The motivation for the present work is two-fold: firstly, we want to show how the contributions of isolated periodic orbits in terms of the $4 \times 4$-stability matrix can be obtained directly from a boundary integral equation without involving large matrices. Secondly, we want to present a practical method for the determination of semiclassical contributions of isolated periodic orbits and families of orbits in systems with axial symmetry, including the determination of the stability matrix and the Maslov index. Furthermore, the method is convenient for the treatment of more complex contributions of periodic orbits like those of bifurcations that are of interest in nuclear physics applications [21].

\section{The boundary integral equation}

A powerful method for investigating quantum billiards, numerically as well as analytically, consists in replacing the Schrödinger equation and boundary conditions by an integral equation. This integral equation is defined on the billiard boundary and effectively reduces the dimension of the problem by one. For three-dimensional billiards with Dirichlet boundary conditions the integral equation has the following form 22

$$
-\int_{\partial \mathcal{D}} d \sigma \partial_{\hat{n}^{\prime}} G_{0}\left(\vec{r}, \vec{r}^{\prime}, E\right) \partial_{\hat{n}} \psi(\vec{r})=\frac{1}{2} \partial_{\hat{n}^{\prime}} \psi\left(\vec{r}^{\prime}\right) .
$$


Here $\mathcal{D}$ denotes the three-dimensional domain of the billiard system, and $\partial \mathcal{D}$ is the boundary surface. For simplicity we assume in the following that the domain $\mathcal{D}$ is compact and the boundary $\partial \mathcal{D}$ is $\mathcal{C}^{2}$. The vectors $\vec{r}$ and $\vec{r}^{\prime}$ are located on the boundary $\partial \mathcal{D}$, and $d \sigma$ is an area element at $\vec{r}$. Furthermore, $\hat{n}$ is a unit normal vector to the boundary pointing outwards, and $\partial_{\hat{n}}=\hat{n} \cdot \vec{\nabla}$ is the component of the gradient in direction of $\hat{n}$. Dimensionless units with $\hbar=2 m=1$ are used throughout, $\psi$ is a solution of the Schrödinger equation with Dirichlet boundary conditions, and $G_{0}$ denotes the free outgoing Green function which is given by

$$
G_{0}\left(\vec{r}, \vec{r}^{\prime}, E\right)=-\frac{\exp \left\{i k\left|\vec{r}-\vec{r}^{\prime}\right|\right\}}{4 \pi\left|\vec{r}-\vec{r}^{\prime}\right|},
$$

where $k=\sqrt{E}$. Eq. (1) is a Fredholm equation of the second kind. It has non-trivial solutions only if the determinant $\Delta(E):=\operatorname{det}(\mathbf{1}-\hat{Q}(E))$ vanishes, where $\hat{Q}$ is the integral operator defined on the boundary $\partial \mathcal{D}$ by

$$
\hat{Q} u\left(\vec{r}^{\prime}\right)=-2 \int_{\partial \mathcal{D}} d \sigma \partial_{\hat{n}^{\prime}} G_{0}\left(\vec{r}, \vec{r}^{\prime}, E\right) u(\vec{r}) .
$$

In recent years there were several publications on the Fredholm determinant $\Delta(E)$ for twodimensional billiards. The integral equation in two dimensions is non-singular if the boundary is continuously differentiable, i. e. if it doesn't have corners or cusps (see [23, 24]). For the following discussion we assume that $\mathcal{D}$ is compact and simply connected, and that the boundary $\partial \mathcal{D}$ is $\mathcal{C}^{2}$, although the following results are valid under less restrictive conditions. By an application of Fredholm theory, $\Delta(E)$ can be represented by an absolutely convergent series which, when approximated semiclassically, yields a series in terms of so-called pseudo-orbits [25, 19, 26]. Considered as a function of $k$ the determinant $\Delta(E)$ is holomorphic in the $k$ plane except for a cut which is due to the cut of the free outgoing Green function in two dimensions. It is known that in the upper half plane $\operatorname{Im} k \geq 0$ the determinant has only zeros which correspond to the eigenvalues of the Schrödinger equation for the interior billiard with Dirichlet boundary conditions $k= \pm \sqrt{E_{n}}$. For $\operatorname{Im} k<0$ there can be further zeros. These additional zeros correspond to the resonances of the outside scattering problem with Neumann boundary conditions [27, 28].

The spectral staircase for the interior problem can be represented in the following form

$$
N(E)=N_{\mathrm{sm}}(E)-\frac{1}{\pi} \lim _{\varepsilon \rightarrow 0+} \operatorname{Im} \log \frac{\Delta(E+i \varepsilon)}{\Delta(0)},
$$

where the second term on the right-hand side jumps by one at every eigenvalue of the Schrödinger equation (or by the multiplicity of an eigenvalue if it is degenerate). $N_{\mathrm{sm}}(E)$ is a smooth function given by

$$
N_{\mathrm{sm}}(E)=\frac{1}{\pi} \int_{0}^{E} d x \lim _{\varepsilon \rightarrow 0+} \operatorname{Im} \frac{\Delta^{\prime}}{\Delta}(x+i \varepsilon) .
$$

It has been conjectured in [27] and shown in [29] that $\pi N_{\mathrm{sm}}(E)$ is equal to the total scattering phase $\eta_{\mathrm{N}}(E)=\frac{i}{2} \log \operatorname{det} S_{\mathrm{N}}(E)$ for the outside scattering problem with Neumann boundary conditions. The on-shell scattering matrix for this problem is denoted by $S_{\mathrm{N}}(E)$.

The asymptotic expansions of the smooth parts $\bar{N}(E)$ of the spectral staircase and $\bar{\eta}_{\mathrm{N}}(E) / \pi$ of the total scattering phase agree in the first three leading terms and in general differ in the 
next terms [27]. A relation between the complete asymptotic expansions of the smooth part $\bar{N}(E)$ for the inner billiard problem and of the smooth part $\bar{\eta}(E)$ for the outside scattering problem with the same boundary conditions was conjectured and proved for several leading terms (13 terms for the Dirichlet case) in [30]. It is stated that if the asymptotic expansion of $\bar{N}(E)$ is

$$
\bar{N}(E) \sim a E+b E^{1 / 2}+c+\sum_{n=1}^{\infty} c_{n} E^{1 / 2-n},
$$

then the asymptotic expansion of $\bar{\eta}(E)$ is

$$
\frac{\bar{\eta}(E)}{\pi} \sim a E-b E^{1 / 2}+c-\sum_{n=1}^{\infty} c_{n} E^{1 / 2-n} .
$$

A semiclassical evaluation of the second term on the right-hand side of (4) yields contributions from interior periodic orbits as well as from periodic orbits that run in the exterior of the billiard (if they exist) [19, 28]. The contributions from the exterior orbits have to be cancelled by corresponding contributions contained in $N_{\mathrm{sm}}(E)$. This is demonstrated in [31].

Finally, the spectral determinant can be factorized in the form $\Delta(E)=\Delta(0) D_{\text {int }}(E) D_{\text {ext }}(E)$ where $D_{\text {int }}(E)$ and $D_{\text {ext }}(E)$ are completely specified in terms of the inside and outside billiard problem, respectively [27, 28].

In the present paper we do not aim at a generalization of the two-dimensional results to three dimensions. We rather use the boundary integral equation as starting point for the derivation of semiclassical contributions of periodic orbits. One difference in comparison to the two-dimensional case is that the integral equation in (11) is singular since the integral kernel has a $1 /\left|\vec{r}-\vec{r}^{\prime}\right|$ singularity. In the surface integral (11) this singularity is integrable, but the trace of the integral operator $\hat{Q}$ is infinite, and the Fredholm determinant has to be regularized.

This can be done by considering the parameter dependent spectral determinant

$$
\Delta_{\lambda}(E):=\operatorname{det}(\mathbf{1}-\lambda \hat{Q}(E))=\exp \left\{-\sum_{n=1}^{\infty} \frac{\lambda^{n}}{n} \operatorname{Tr} \hat{Q}^{n}(E)\right\}, \quad|\lambda|<R,
$$

whose representation by the sum over $n$ has a positive radius of convergence $R$ if all traces are finite. If the first $l$ traces $\hat{Q}^{n}(E), n=1, \ldots, l$, are infinite one can define a modified Fredholm determinant by replacing the sum in (8) by a similar expression in which the first $l$ traces are missing [32]. This expression can be analytically continued to the whole complex $\lambda$-plane. The new determinant again has zeros at the eigenvalues of the Schrödinger equation. In case of the integral operator (3) the first two traces $\operatorname{Tr} \hat{Q}$ and $\operatorname{Tr} \hat{Q}^{2}$ are divergent.

\section{The semiclassical Green function}

We take the derivative of eq. (4) with respect to $k$, insert (8) for $\lambda=1$, and obtain the following equation for the level density in terms of the wavenumber $k$

$$
d(k)=d_{\mathrm{sm}, n_{0}}(k)+\frac{1}{\pi} \frac{d}{d k} \operatorname{Im} \sum_{n=n_{0}}^{\infty} \frac{1}{n} \operatorname{Tr} \hat{Q}^{n}(k)
$$


where

$$
\operatorname{Tr} \hat{Q}^{n}(k)=(-2)^{n} \int_{\partial \mathcal{D}} d \sigma_{1} \ldots d \sigma_{n} \partial_{\hat{n}_{1}} G_{0}\left(\vec{r}_{2}, \vec{r}_{1}, E\right) \partial_{\hat{n}_{2}} G_{0}\left(\vec{r}_{3}, \vec{r}_{2}, E\right) \ldots \partial_{\hat{n}_{n}} G_{0}\left(\vec{r}_{1}, \vec{r}_{n}, E\right) .
$$

Here the vectors $\vec{r}_{i}$ are located on the boundary, $d \sigma_{i}$ is an area element at $\vec{r}_{i}$, and $\hat{n}_{i}$ is the outward normal vector at $\vec{r}_{i}$.

As noted in the previous section, all traces of powers of $\hat{Q}$ in the sum in (9) are finite if the sum starts with $n_{0} \geq 3$. For the semiclassical approximation this has the disadvantage that the oscillatory contributions of $n$-bounce orbits with $n<n_{0}$ are shifted into the smooth part $d_{\mathrm{sm}, n_{0}}(k)$ through eq. (5) with the redefined spectral determinant. This problem can be avoided by replacing the integral kernel in (3) by its leading semiclassical term. Then the integral kernel is non-singular and yields the same leading semiclassical contributions to (10) as before. For the derivation of the semiclassical approximation, i. e. after replacing the integral kernel by its leading semiclassical term, $n_{0}$ thus can be set to $n_{0}=1$.

For real values of $k$ a second divergence occurs since the sum in (9) is in general divergent for real $k$. One can avoid this by starting with an exact formula for a smoothed level density [27. We assume for the following derivation that $k$ has a sufficiently large imaginary part in order to make the sum convergent, and that $\operatorname{Re} k>0$.

The integrals that appear in eq. (10) can be given a simple interpretation in terms of the Green function of the billiard system. According to the multiple reflection expansion of Balian and Bloch [14] this Green function can be represented by a sum over partial Green functions $G^{(n)}$ corresponding to $n$ reflections on the billiard boundary, with $0 \leq n<\infty$

$$
G\left(\vec{r}, \vec{r}^{\prime}, E\right)=\sum_{n=0}^{\infty} G^{(n)}\left(\vec{r}, \vec{r}^{\prime}, E\right)
$$

where

$$
G^{(n)}\left(\vec{r}, \vec{r}^{\prime}, E\right)=(-2)^{n} \int_{\partial \mathcal{D}} d \sigma_{1} \ldots d \sigma_{n} G_{0}\left(\vec{r}_{1}, \vec{r}^{\prime}, E\right) \partial_{\hat{n}_{1}} G_{0}\left(\vec{r}_{2}, \vec{r}_{1}, E\right) \ldots \partial_{\hat{n}_{n}} G_{0}\left(\vec{r}, \vec{r}_{n}, E\right) .
$$

A comparison of (10) and (12) shows that

$$
d(k)=d_{\mathrm{sm}, n_{0}}(k)-\left.\frac{2}{\pi} \frac{d}{d k} \operatorname{Im} \sum_{n=n_{0}}^{\infty} \frac{1}{n} \int_{\partial \mathcal{D}} d \sigma \partial_{\hat{n}^{\prime}} G^{(n-1)}\left(\vec{r}, \vec{r}^{\prime}, E\right)\right|_{\vec{r}=\vec{r}^{\prime}} .
$$

In the following we derive semiclassical contributions of periodic orbits to $d(k)$ by evaluating the integrals in (12) and (13) in stationary phase approximation. The semiclassical evaluation of (12) expresses the partial Green function $G^{(n)}$ in terms of classical trajectories from $\vec{r}^{\prime}$ to $\vec{r}$. These trajectories can be of three kinds: interior orbits which run inside the billiard domain $\mathcal{D}$, exterior orbits which run outside $\mathcal{D}$, and ghost orbits which cross the boundary and run inside and outside. However, in the sum (11) the leading order contributions of ghost orbits from different $n$ cancel [16]. In the following we restrict to the consideration of interior orbits, i. e. we denote by $G_{\mathrm{sc}}^{(n)}$ the semiclassical contributions to $G^{(n)}$ from interior orbits. We will show that this contribution is given by

$$
G_{\mathrm{sc}}^{(n)}\left(\vec{r}, \vec{r}^{\prime}, E\right)=\sum_{\gamma_{n}} \frac{1}{4 \pi \sqrt{\left|k^{2} \operatorname{det} B_{\gamma_{n}}\right|}} \exp \left\{i k l_{\gamma_{n}}-i \frac{\pi}{2} \nu_{\gamma_{n}}-i \pi\right\}
$$


where $\gamma_{n}$ labels all trajectories that run from $\vec{r}^{\prime}$ to $\vec{r}$ and are reflected $n$ times on the boundary in between. Furthermore, $l_{\gamma_{n}}$ denotes the length of the trajectory $\gamma_{n}$, and $\nu_{\gamma_{n}}$ is the number of conjugate points from $\vec{r}^{\prime}$ to $\vec{r}$ plus twice the number $n$ of reflections on the boundary. $B_{\gamma_{n}}$ is a submatrix of the stability matrix for the trajectory. The stability matrix is defined by

$$
\left(\begin{array}{l}
\mathrm{d} \vec{q}_{\perp} \\
\mathrm{d} \vec{p}_{\perp}
\end{array}\right)=M\left(\begin{array}{l}
\mathrm{d} \vec{q}_{\perp}^{\prime} \\
\mathrm{d} \vec{p}_{\perp}^{\prime}
\end{array}\right), \quad M=\left(\begin{array}{cc}
A & B \\
C & D
\end{array}\right) .
$$

Here $\mathrm{d} \vec{q}_{\perp}{ }^{\prime}$ and $\mathrm{d} \vec{p}_{\perp}{ }^{\prime}$ are two-dimensional vectors which describe infinitesimal, perpendicular deviations from a considered trajectory at the starting point. $\mathrm{d} \vec{q}_{\perp}$ and $\mathrm{d} \vec{p}_{\perp}$ are the corresponding deviations at the end point that are obtained by a linearization of the motion in the vicinity of the considered trajectory. Correspondingly, $M$ is a $4 \times 4$-matrix, and $A, B, C$ and $D$ are $2 \times 2$ matrices. The determinant of the submatrix $B$ is thus given by $\operatorname{det} B=M_{13} M_{24}-M_{14} M_{23}$. The denominator in (14) is independent of $k$ since $\operatorname{det} B$ is proportional to $1 / E$. Properties of the symplectic stability matrix are given in appendix $\mathrm{A}$, and a method for its determination is described in appendix $\mathrm{B}$.

We prove now eq. (14) by induction. For $n=0$ it is correct since

$$
G_{0}\left(\vec{r}, \vec{r}^{\prime}, E\right)=G_{\mathrm{sc}}^{(0)}\left(\vec{r}, \vec{r}^{\prime}, E\right) .
$$

This follows from the form of $G_{0}$ in (2) and the fact that $\nu=0$ and $\operatorname{det} B=\left(\vec{r}-\vec{r}^{\prime}\right)^{2}$ for the free motion (see the stability matrix for the free motion in appendix B).

It remains to show that

$$
(-2) \int_{\partial \mathcal{D}} d \sigma G_{\mathrm{sc}}^{(n)}\left(\vec{r}, \vec{r}_{a}, E\right) \partial_{\hat{n}} G_{0}\left(\vec{r}_{b}, \vec{r}, E\right) \approx G_{\mathrm{sc}}^{(n+1)}\left(\vec{r}_{b}, \vec{r}_{a}, E\right),
$$

where the approximate sign denotes that the integral is evaluated in stationary phase approximation. We use the following notation at the point $\vec{r}$ of the boundary: The momentum of the particle for the incoming trajectory is denoted by $\vec{p}^{\prime}$, and the momentum of the outgoing trajectory is $\vec{p}$. Both have modulus $k=\sqrt{E}$.

The normal derivative of the Green function is given in leading semiclassical order by

$$
\partial_{\hat{n}} G_{0}\left(\vec{r}_{b}, \vec{r}, E\right) \approx-i \hat{n} \cdot \vec{p} G_{\mathrm{sc}}^{(0)}\left(\vec{r}_{b}, \vec{r}, E\right)=i k \cos \alpha G_{\mathrm{sc}}^{(0)}\left(\vec{r}_{b}, \vec{r}, E\right),
$$

where $\alpha$ is the angle between $-\hat{n}$ and $\vec{p}$. The left-hand side of (17) thus is a sum over terms of the form

$$
-2 i k \cos \alpha \int_{\partial \mathcal{D}} d s_{1} d s_{2} \frac{\exp \left\{i k\left[l^{(n)}\left(\vec{r}, \vec{r}_{a}\right)+l^{(0)}\left(\vec{r}_{b}, \vec{r}\right)\right]-i \frac{\pi}{2} \nu^{(n)}\right\}}{16 \pi^{2} l^{(0)} \sqrt{\left|k^{2} \operatorname{det} B^{(n)}\right|}}
$$

where the area element $d \sigma$ has been replaced by $d s_{1} d s_{2}$.

The stationary points of the above integral are determined by the conditions

$$
0=\frac{d}{d s_{1,2}}\left[l^{(0)}\left(\vec{r}_{b}, \vec{r}\right)+l^{(n)}\left(\vec{r}, \vec{r}_{a}\right)\right]=\hat{t}_{1,2} \cdot\left[-\frac{\vec{p}}{k}+\frac{\vec{p}^{\prime}}{k}\right],
$$

where $t_{1}$ and $t_{2}$ are two orthogonal unit tangential vectors at the considered point of reflection. In eq. (19) and in the following the length of a trajectory is given two arguments when it is necessary to specify the start and the end point of a trajectory. The second equation in (20) follows from the relation of the length function to the action $S\left(\vec{r}, \vec{r}^{\prime}, E\right)=k l\left(\vec{r}, \vec{r}^{\prime}\right)$. 
We neglect the possible solution $\vec{p}=\vec{p}^{\prime}$ of $(20)$ since it leads to contributions of ghost orbits. The other solution of (20) yields the condition for elastic reflection: $\vec{p}, \vec{p}^{\prime}$ and $\hat{n}$ lie in one plane, the reflection plane, and the angles between $-\vec{p}$ and $\hat{n}$ and between $\hat{n}$ and $\vec{p}^{\prime}$ are identical. The value of this angle $\alpha$ lies in the range $0 \leq \alpha<\pi / 2$. The sum over all stationary points thus expresses the integral $I$ by a sum over all trajectories with $n+1$ reflections on the boundary.

For the following evaluations it will be useful to express the tangential vectors and the normal vector in local coordinate systems of the trajectory, in which the first coordinate is always in direction of the trajectory, and the other two coordinates are perpendicular to it.

$$
\begin{aligned}
& \hat{n}=-\cos \alpha \hat{e}_{1}-\sin \alpha \hat{e}_{2}=\cos \alpha \hat{e}_{1}^{\prime}-\sin \alpha \hat{e}_{2}^{\prime}=\cos \alpha \hat{e}_{1}^{\prime \prime}-\sin \alpha\left[\cos \theta \hat{e}_{2}^{\prime \prime}+\sin \theta \hat{e}_{3}^{\prime \prime}\right], \\
& \hat{t}_{1}=\sin \alpha \hat{e}_{1}-\cos \alpha \hat{e}_{2}=\sin \alpha \hat{e}_{1}^{\prime}+\cos \alpha \hat{e}_{2}^{\prime}=\sin \alpha \hat{e}_{1}^{\prime \prime}+\cos \alpha\left[\cos \theta \hat{e}_{2}^{\prime \prime}+\sin \theta \hat{e}_{3}^{\prime \prime}\right] \text {, } \\
& \hat{t}_{2}=\hat{e}_{3} \quad=\hat{e}_{3}^{\prime} \quad=\cos \theta \hat{e}_{3}^{\prime \prime}-\sin \theta \hat{e}_{2}^{\prime \prime} \text {, }
\end{aligned}
$$

where $\hat{t}_{2}=\hat{n} \times \hat{t}_{1}$. In (21) the unit vectors $\hat{e}_{i}^{\prime \prime}$ belong to the local coordinate system for the incoming trajectory, and the unit vector $\hat{e}_{2}^{\prime \prime}$ lies in the reflection plane of the previous reflection. This coordinate system is rotated around the trajectory such that the new vector $\hat{e}_{2}^{\prime}$ lies in the new reflection plane. The unprimed coordinate system is the local coordinate system directly after the reflection, and $\hat{e}_{2}$ again lies in the reflection plane.

We continue now with the evaluation of the integral in eq. (19) by stationary phase approximation. For that purpose the second derivatives of the exponent have to be determined. According to (20) they consist of two parts, the derivatives of the tangential vectors and the derivatives of the momenta. Those of the tangential vectors follow from eq. (60) of appendix D and are given by

$$
\frac{\partial \hat{t}_{1}}{\partial s_{1}}=-\frac{\hat{n}}{R_{a}}, \quad \frac{\partial \hat{t}_{1}}{\partial s_{2}}=-\frac{\hat{n}}{R_{c}}, \quad \frac{\partial \hat{t}_{2}}{\partial s_{1}}=-\frac{\hat{n}}{R_{c}}, \quad \frac{\partial \hat{t}_{2}}{\partial s_{2}}=-\frac{\hat{n}}{R_{b}},
$$

where $R_{a}, R_{b}$ are the radii of curvature of the surface in the reflection plane and perpendicular to it, respectively. $R_{a}, R_{b}$ and $R_{c}$ are given in (50) in terms of the two main radii of curvature $R_{1}$ and $R_{2}$ and the angle $\beta$ between $\hat{t}_{1}$ and the main curvature direction for $R_{1}$.

The derivatives of the momenta of the incoming and outgoing trajectory are expressed in terms of the local coordinate systems before and after the reflection, respectively.

$$
\begin{array}{lll}
\frac{\partial \vec{p}}{\partial s_{1}}=\left(\hat{t}_{1} \cdot \vec{\nabla}\right) \vec{p}=-\cos \alpha\left(\frac{\partial p_{2}}{\partial q_{2}} \hat{e}_{2}+\frac{\partial p_{3}}{\partial q_{2}} \hat{e}_{3}\right), & \frac{\partial \vec{p}}{\partial s_{2}}=\left(\hat{t}_{2} \cdot \vec{\nabla}\right) \vec{p}=\frac{\partial p_{2}}{\partial q_{3}} \hat{e}_{2}+\frac{\partial p_{3}}{\partial q_{3}} \hat{e}_{3}, \\
\frac{\partial \vec{p}^{\prime}}{\partial s_{1}}=\left(\hat{t}_{1} \cdot \vec{\nabla}\right) \vec{p}^{\prime}=\cos \alpha\left(\frac{\partial p_{2}^{\prime}}{\partial q_{2}^{\prime}} \hat{e}_{2}^{\prime}+\frac{\partial p_{3}^{\prime}}{\partial q_{2}^{\prime}} \hat{e}_{3}^{\prime}\right), & \frac{\partial \vec{p}^{\prime}}{\partial s_{2}}=\left(\hat{t}_{2} \cdot \vec{\nabla}\right) \vec{p}^{\prime}=\frac{\partial p_{2}^{\prime}}{\partial q_{3}^{\prime}} \hat{e}_{2}^{\prime}+\frac{\partial p_{3}^{\prime}}{\partial q_{3}^{\prime}} \hat{e}_{3}^{\prime},
\end{array}
$$

and with $(20),(22)$ and (23) the second derivatives of the length function follow as

$$
\begin{aligned}
& a_{1}:=\frac{\partial^{2}}{\partial s_{1} \partial s_{1}}\left[l^{(0)}\left(\vec{r}_{b}, \vec{r}\right)+l^{(n)}\left(\vec{r}, \vec{r}_{a}\right)\right]=-\frac{2 \cos \alpha}{R_{a}}+\left[-\frac{\partial p_{2}}{\partial q_{2}}+\frac{\partial p_{2}^{\prime}}{\partial q_{2}^{\prime}}\right] \frac{\cos ^{2} \alpha}{k}, \\
& b_{1}:=\frac{\partial^{2}}{\partial s_{2} \partial s_{1}}\left[l^{(0)}\left(\vec{r}_{b}, \vec{r}\right)+l^{(n)}\left(\vec{r}, \vec{r}_{a}\right)\right]=-\frac{2 \cos \alpha}{R_{c}}+\left[\frac{\partial p_{2}}{\partial q_{3}}+\frac{\partial p_{2}^{\prime}}{\partial q_{3}^{\prime}}\right] \frac{\cos \alpha}{k} \\
& c_{1}:=\frac{\partial^{2}}{\partial s_{1} \partial s_{2}}\left[l^{(0)}\left(\vec{r}_{b}, \vec{r}\right)+l^{(n)}\left(\vec{r}, \vec{r}_{a}\right)\right]=-\frac{2 \cos \alpha}{R_{c}}+\left[\frac{\partial p_{3}}{\partial q_{2}}+\frac{\partial p_{3}^{\prime}}{\partial q_{2}^{\prime}}\right] \frac{\cos \alpha}{k} \\
& d_{1}:=\frac{\partial^{2}}{\partial s_{2} \partial s_{2}}\left[l^{(0)}\left(\vec{r}_{b}, \vec{r}\right)+l^{(n)}\left(\vec{r}, \vec{r}_{a}\right)\right]=-\frac{2 \cos \alpha}{R_{b}}+\left[-\frac{\partial p_{3}}{\partial q_{3}}+\frac{\partial p_{3}^{\prime}}{\partial q_{3}^{\prime}}\right] \frac{1}{k}
\end{aligned}
$$


We discuss in more detail how the partial derivatives of the momenta in (24) have to be interpreted. The derivatives $\partial p_{i} / \partial q_{j}$ describe the change of the component $p_{i}$ upon a change of $q_{j}$ when the other components of $\vec{q}$ and $\vec{q}_{b}$ are hold fixed. Thus it can be recognized as an element of the $N$-matrix of appendix $\mathrm{A}$ for the free motion from $\vec{q}$ to $\vec{q}_{b}$. By the relations of appendix A it can be expressed in terms of the stability matrix $M_{T}$ for this part of the trajectory. Note, however, that primed and unprimed quantities have a different meaning here and in appendix A. Here the unprimed quantities are the quantities directly after the reflection, i. e. they describe the beginning of the trajectory from $\vec{q}$ to $\vec{q}_{b}$. In the appendix $\mathrm{A}$, however, the unprimed quantities are those at the end of a trajectory, so the meaning is reversed.

The derivatives $\partial p_{i}^{\prime} / \partial q_{j}^{\prime}$ on the other hand describe the change of the component $p_{i}^{\prime}$ upon a change of $q_{j}^{\prime}$ when the other components of $\vec{q}^{\prime}$ and $\vec{q}_{a}$ are hold fixed. Again these partial derivatives are elements of an $N$-matrix, but now for the trajectory from $\vec{q}_{a}$ to $\vec{q}^{\prime}$, including the final rotation that transforms the double primed coordinate system into the primed one. Again by the relations of appendix A they can be expressed in terms of the stability matrix $M^{\prime}=M_{S} M^{(n)}$, where the rotation matrix $M_{S}$ is given in (48).

The quantities $a_{1}, \ldots, d_{1}$ in (24) can also be expressed in terms of the stability matrix for the full trajectory from $\vec{q}_{a}$ to $\overrightarrow{q_{b}}$, that is given by $M:=M^{(n+1)}=M_{T} M_{R} M_{S} M^{(n)}$ where $M_{R}$ is the stability matrix for a reflection given in (49). The following expressions can be proved by a direct although lengthy evaluation that was done with Maple. One finds that

$$
\begin{array}{rlrl}
a_{1} & =-\frac{\left[M_{13} M_{24}^{\prime}-M_{14} M_{23}^{\prime}\right]}{\left[M_{13}^{\prime} M_{24}^{\prime}-M_{14}^{\prime} M_{23}^{\prime}\right]} \frac{\cos ^{2} \alpha}{l^{(0)}}, & b_{1} & =\frac{\left[M_{13} M_{14}^{\prime}-M_{14} M_{13}^{\prime}\right]}{\left[M_{13}^{\prime} M_{24}^{\prime}-M_{14}^{\prime} M_{23}^{\prime}\right]} \frac{\cos \alpha}{l^{(0)}}, \\
c_{1}=\frac{\left[M_{23} M_{24}^{\prime}-M_{24} M_{23}^{\prime}\right]}{\left[M_{13}^{\prime} M_{24}^{\prime}-M_{14}^{\prime} M_{23}^{\prime}\right]} \frac{\cos \alpha}{l^{(0)}}, & d_{1}=-\frac{\left[M_{23} M_{14}^{\prime}-M_{24} M_{13}^{\prime}\right]}{\left[M_{13}^{\prime} M_{24}^{\prime}-M_{14}^{\prime} M_{23}^{\prime}\right]} \frac{1}{l^{(0)}} .
\end{array}
$$

and

$$
a_{1} d_{1}-b_{1} c_{1}=-\frac{\left[M_{13} M_{24}-M_{14} M_{23}\right]}{\left[M_{13}^{\prime} M_{24}^{\prime}-M_{14}^{\prime} M_{23}^{\prime}\right]} \frac{\cos ^{2} \alpha}{\left(l^{(0)}\right)^{2}}=-\frac{\cos ^{2} \alpha \operatorname{det} B}{\left(l^{(0)}\right)^{2} \operatorname{det} B^{\prime}},
$$

where $M=M_{T} M_{R} M^{\prime}$ and $M^{\prime}=M_{S} M^{(n)}$ as before. With (26) the contribution of a stationary point to the integral in $(\sqrt{19})$ can be evaluated and results in

$$
\begin{aligned}
I_{\mathrm{sp}} & =-i k \cos \alpha \int d s_{1} d s_{2} \frac{\exp \left\{i k\left[l^{(n)}+l^{(0)}\right]+\frac{i k}{2}\left[a_{1} s_{1}^{2}+\left(b_{1}+c_{1}\right) s_{1} s_{2}+d_{1} s_{2}^{2}\right]-i \frac{\pi}{2} \nu^{(n)}\right\}}{8 \pi^{2} l^{(0)} \sqrt{\left|k^{2} \operatorname{det} B^{\prime}\right|}} \\
& =\frac{\exp \left\{i k l^{(n+1)}-i \frac{\pi}{2} \nu^{(n+1)}-i \pi\right\}}{4 \pi \sqrt{\mid k^{2} \operatorname{det} B^{(n+1) \mid}}},
\end{aligned}
$$

where $\operatorname{det} B^{\prime}=\operatorname{det} B^{(n)}$ has been used, and

$$
\nu^{(n+1)}=\nu^{(n)}+2+ \begin{cases}0 & \text { if } a_{1}>0 \text { and }\left(a_{1} d_{1}-b_{1} c_{1}\right)>0 \\ 1 & \text { if }\left(a_{1} d_{1}-b_{1} c_{1}\right)<0 \\ 2 & \text { if } a_{1}<0 \text { and }\left(a_{1} d_{1}-b_{1} c_{1}\right)>0 .\end{cases}
$$

The expression (27) has the correct form of a contribution to the semiclassical Green function in (14). It remains to show that the condition (28) gives the correct index $\nu^{(n+1)}$ that is defined as the number of conjugate points from $\vec{r}_{a}$ to $\vec{r}_{b}$ plus twice the number of reflections in between. 
The index $\nu^{(n+1)}$ increases in comparison to $\nu^{(n)}$ by 2 because of the additional reflection, and possibly further by 0,1 or 2 , depending on the number of conjugate points between $\vec{r}$ and $\vec{r}_{b}$. To determine these conjugate points we consider $\operatorname{det} B$ as a function of the length $l^{(0)}$ as we vary the end point of the trajectory from $\vec{r}$ to $\vec{r}_{b}$. A conjugate point occurs at every zero of $\operatorname{det} B$, and there can be at most two zeros between $\vec{r}$ to $\vec{r}_{b}$, because $\operatorname{det} B$ is a quadratic function of $l^{(0)}$. This follows from the form of the matrix for free motion $M_{T}$ in (48). We note that a multiplication of a stability matrix by a reflection matrix $M_{R}$ changes only the sign of $\operatorname{det} B$.

From these considerations follows that there is exactly one zero of $\operatorname{det} B$ between $\vec{r}$ and $\vec{r}_{b}$ if $\left(a_{1} d_{1}-b_{1} c_{1}\right)<0$ because then det $B$ has changed sign between $\vec{r}$ and $\vec{r}_{b}$. If $\left(a_{1} d_{1}-b_{1} c_{1}\right)>0$ there can be no or two zeros of $\operatorname{det} B$ between $\vec{r}$ and $\vec{r}_{b}$. This depends on the sign of $a_{1}$. The quantity $a_{1}$ has at most one zero as a function of $l^{(0)}$ as can be seen from its dependence on $l^{(0)}$ in

$$
a_{1}=\frac{\cos ^{2} \alpha}{l^{(0)}}+e_{1}, \quad b_{1}=e_{2}, \quad c_{1}=e_{2}, \quad d_{1}=\frac{1}{l^{(0)}}+e_{3},
$$

where $e_{1}, e_{2}$ and $e_{3}$ are independent of $l^{(0)}$. Furthermore, the dependence of the coefficient $a_{1}, \ldots, d_{1}$ on $l^{(0)}$ shows that a zero of $a_{1}$ is always after a first zero of $\operatorname{det} B$, and before a second zero of det $B$ there is always a zero of $a_{1}$. (If $a_{1}=0$ then $\left(a_{1} d_{1}-b_{1} c_{1}\right)<0$ and for that reason there must be a zero of $\left(a_{1} d_{1}-b_{1} c_{1}\right)$ before since both, $a_{1}$ and $\left(a_{1} d_{1}-b_{1} c_{1}\right)$, are positive for small $l^{(0)}$. Furthermore, $a_{1}$ and $d_{1}$ are monotonously decreasing functions of $l^{(0)}$. If $a_{1} d_{1}=e_{2}^{2}$ for two different values of $l^{(0)}$, both, $a_{1}$ and $d_{1}$, must have changed sign in between.) Thus the number of conjugate points is given correctly by (28) and the semiclassical expression for $G^{(n)}$ in (14) is correct.

\section{Contributions of periodic orbits}

We continue with the evaluation of the integral (13) for the spectral density. Inserting the semiclassical expressions (18) and (14) for the partial Green function into (13) yields contributions to the level density of the form

$$
\frac{2}{\pi} \frac{d}{d k} \operatorname{Im} \frac{1}{n} \int_{\partial \mathcal{D}} d s_{1} d s_{2} i k \cos \alpha \frac{\exp \left\{i k l^{(n-1)}-i \frac{\pi}{2} \nu^{(n-1)}\right\}}{4 \pi \sqrt{\left|k^{2} \operatorname{det} B^{(n-1)}\right|}} .
$$

We consider now the contributions of stationary points to the integral in (30). The stationary points are determined by

$$
0=\frac{d}{d s_{1,2}}\left[l^{(n-1)}(\vec{r}, \vec{r})\right]=\hat{t}_{1,2} \cdot\left[-\frac{\vec{p}}{k}+\frac{\vec{p}^{\prime}}{k}\right]
$$

which is the condition for a specular reflection. Again $\vec{p}^{\prime}$ is the momentum directly before the reflection, and $\vec{p}$ the momentum directly after the reflection. The contributions thus come from periodic orbits with $n$ specular reflections on the billiard wall. We consider one such orbit and label it by $\gamma$. It has $n / r_{\gamma}$ distinct reflections on the boundary if $r_{\gamma}$ is its repetition number. Consequently, one obtains $n / r_{\gamma}$ identical semiclassical contributions for this orbit, since $n / r_{\gamma}$ different stationary points correspond to it. (That the contributions are identical follows from 
the fact that a stationary phase contribution to $\operatorname{Tr} \hat{Q}^{n}$ in (10) does not depend on the order in which the $n$ integrals are evaluated.)

For a determination of the second derivatives of the exponent in (30) the derivatives of the momenta $\vec{p}$ and $\vec{p}^{\prime}$ are needed.

$$
\begin{aligned}
& \frac{\partial \vec{p}}{\partial s_{1}}=\left(\hat{t}_{1} \cdot \vec{\nabla}\right) \vec{p}=\left(-\frac{\partial p_{2}}{\partial q_{2}} \hat{e}_{2}-\frac{\partial p_{3}}{\partial q_{2}} \hat{e}_{3}+\frac{\partial p_{2}}{\partial q_{2}^{\prime}} \hat{e}_{2}+\frac{\partial p_{3}}{\partial q_{2}^{\prime}} \hat{e}_{3}\right) \cos \alpha, \\
& \frac{\partial \vec{p}}{\partial s_{2}}=\left(\hat{t}_{2} \cdot \vec{\nabla}\right) \vec{p}=\frac{\partial p_{2}}{\partial q_{3}} \hat{e}_{2}+\frac{\partial p_{3}}{\partial q_{3}} \hat{e}_{3}+\frac{\partial p_{2}}{\partial q_{3}^{\prime}} \hat{e}_{2}+\frac{\partial p_{3}}{\partial q_{3}^{\prime}} \hat{e}_{3}, \\
& \frac{\partial \vec{p}^{\prime}}{\partial s_{1}}=\left(\hat{t}_{1} \cdot \vec{\nabla}\right) \vec{p}^{\prime}=\left(-\frac{\partial p_{2}^{\prime}}{\partial q_{2}} \hat{e}_{2}^{\prime}-\frac{\partial p_{3}^{\prime}}{\partial q_{2}} \hat{e}_{3}^{\prime}+\frac{\partial p_{2}^{\prime}}{\partial q_{2}^{\prime}} \hat{e}_{2}^{\prime}+\frac{\partial p_{3}^{\prime}}{\partial q_{2}^{\prime}} \hat{e}_{3}^{\prime}\right) \cos \alpha, \\
& \frac{\partial \vec{p}^{\prime}}{\partial s_{2}}=\left(\hat{t}_{2} \cdot \vec{\nabla}\right) \vec{p}^{\prime}=\frac{\partial p_{2}^{\prime}}{\partial q_{3}} \hat{e}_{2}^{\prime}+\frac{\partial p_{3}^{\prime}}{\partial q_{3}} \hat{e}_{3}^{\prime}+\frac{\partial p_{2}^{\prime}}{\partial q_{3}^{\prime}} \hat{e}_{2}^{\prime}+\frac{\partial p_{3}^{\prime}}{\partial q_{3}^{\prime}} \hat{e}_{3}^{\prime} .
\end{aligned}
$$

In contrast to the results in (23) there are additional terms in (32). They arise because a change of the reflection point changes the initial point as well as the end point of the trajectory from $\vec{r}$ to $\vec{r}$. With (31), (22) and (32) we obtain the following expressions

$$
\begin{aligned}
a_{2} & :=\frac{\partial^{2}}{\partial s_{1}^{2}}\left[l^{(n-1)}(\vec{r}, \vec{r})\right]=-\frac{2 \cos \alpha}{R_{a}}+\left[-\frac{\partial p_{2}}{\partial q_{2}}+\frac{\partial p_{2}}{\partial q_{2}^{\prime}}-\frac{\partial p_{2}^{\prime}}{\partial q_{2}}+\frac{\partial p_{2}^{\prime}}{\partial q_{2}^{\prime}}\right] \frac{\cos ^{2} \alpha}{k}, \\
b_{2} & :=\frac{\partial^{2}}{\partial s_{2} \partial s_{1}}\left[l^{(n-1)}(\vec{r}, \vec{r})\right]=-\frac{2 \cos \alpha}{R_{c}}+\left[\frac{\partial p_{2}}{\partial q_{3}}+\frac{\partial p_{2}}{\partial q_{3}^{\prime}}+\frac{\partial p_{2}^{\prime}}{\partial q_{3}}+\frac{\partial p_{2}^{\prime}}{\partial q_{3}^{\prime}}\right] \frac{\cos \alpha}{k}, \\
c_{2} & :=\frac{\partial^{2}}{\partial s_{1} \partial s_{2}}\left[l^{(n-1)}(\vec{r}, \vec{r})\right]=-\frac{2 \cos \alpha}{R_{c}}+\left[\frac{\partial p_{3}}{\partial q_{2}}-\frac{\partial p_{3}}{\partial q_{2}^{\prime}}-\frac{\partial p_{3}^{\prime}}{\partial q_{2}}+\frac{\partial p_{3}^{\prime}}{\partial q_{2}^{\prime}}\right] \frac{\cos \alpha}{k}, \\
d_{2} & :=\frac{\partial^{2}}{\partial s_{2}^{2}}\left[l^{(n-1)}(\vec{r}, \vec{r})\right]=-\frac{2 \cos \alpha}{R_{b}}+\left[-\frac{\partial p_{3}}{\partial q_{3}}-\frac{\partial p_{3}}{\partial q_{3}^{\prime}}+\frac{\partial p_{3}^{\prime}}{\partial q_{3}}+\frac{\partial p_{3}^{\prime}}{\partial q_{3}^{\prime}}\right] \frac{1}{k} .
\end{aligned}
$$

Again all partial derivatives of the momenta are elements of an $N$-matrix that corresponds to the stability matrix $M^{\prime}=M_{S} M^{(n-1)}$, and thus the quantities $a_{2}, \ldots, d_{2}$ can be expressed in terms of the elements of $M^{\prime}$. They can further be expressed in terms of the full stability matrix of the orbit $M=M_{\gamma}=M_{R} M^{\prime}$, where $M_{R}$ describes the reflection. One finds that

$$
\begin{aligned}
a_{2} & =\frac{\mathcal{M}_{42}}{k \operatorname{det} B} \cos ^{2} \alpha, & b_{2} & =-\frac{\mathcal{M}_{41}}{k \operatorname{det} B} \cos \alpha, \\
c_{2} & =-\frac{\mathcal{M}_{32}}{k \operatorname{det} B} \cos \alpha, & d_{2} & =\frac{\mathcal{M}_{31}}{k \operatorname{det} B},
\end{aligned}
$$

and

$$
a_{2} d_{2}-b_{2} c_{2}=\frac{\operatorname{det}(M-1) \cos ^{2} \alpha}{k^{2} \operatorname{det} B} .
$$

The quantity $\mathcal{M}_{i j}$ in (34) is the determinant of the $3 \times 3$-matrix that is obtained by dropping the $i$-th row and the $j$-th column of the matrix $(M-1)$.

With these expressions a stationary phase approximation for the contribution of an isolated periodic orbit results in

$$
\begin{aligned}
d_{\gamma}(k) & =-\frac{l^{(n-1)} \cos \alpha}{2 \pi^{2} r_{\gamma}} \operatorname{Im} \int d s_{1} d s_{2} \frac{k \exp \left\{i k l^{(n-1)}+\frac{i k}{2}\left(a_{2} s_{1}^{2}+\left(b_{2}+c_{2}\right) s_{1} s_{2}+d_{2} s_{2}^{2}\right)-i \frac{\pi}{2} \nu^{(n-1)}\right\}}{\sqrt{\mid k^{2} \operatorname{det} B^{(n-1) \mid}}} \\
& =\frac{l_{\gamma}}{\pi r_{\gamma} \sqrt{\left|\operatorname{det}\left(M_{\gamma}-1\right)\right|}} \operatorname{Rexp}\left\{i k l_{\gamma}-\frac{i \pi}{2} \mu_{\gamma}\right\}
\end{aligned}
$$


where the relations $\left|\operatorname{det} B^{(n-1)}\right|=|\operatorname{det} B|, l_{\gamma}=l^{(n-1)}$ and

$$
\mu_{\gamma}=\nu^{(n-1)}+2+ \begin{cases}0 & \text { if } a_{2}>0 \text { and }\left(a_{2} d_{2}-b_{2} c_{2}\right)>0 \\ 1 & \text { if }\left(a_{2} d_{2}-b_{2} c_{2}\right)<0 \\ 2 & \text { if } a_{2}<0 \text { and }\left(a_{2} d_{2}-b_{2} c_{2}\right)>0\end{cases}
$$

have been used. Eq. (36) is the expected contribution of an isolated periodic orbit. The relations for determining the Maslov index $\mu$ of a periodic orbit are summarized in appendix Q. The conditions for the additional contributions to $\mu_{\gamma}$ in (37) can be shown to be identical to the general conditions in 33 .

In applications three-dimensional billiard systems often have an axial symmetry. Then periodic orbits appear in one-parameter families. In the following we derive the contribution of such a family. A general treatment of semiclassical trace formulas in the presence of continuous symmetries can be found in [34. In this article, a general formula is given for families of orbits in axially symmetric systems. The formula which is obtained in the following has a different form which is not explicitly independent of the starting point of the trajectory, but it has the advantage that it is expressed directly in terms of the stability matrix $M$. Of course, both formulas must yield the same result.

In presence of an axial symmetry the quantity $\left(a_{2} d_{2}-b_{2} c_{2}\right)$ vanishes and the symmetric matrix $\left(\begin{array}{ll}a_{2} & b_{2} \\ c_{2} & d_{2}\end{array}\right)$ has eigenvalues 0 and $\left(a_{2}+d_{2}\right)$. By a rotation of the coordinate system the matrix can be diagonalized and one obtains

$$
\begin{aligned}
d_{\gamma}(k) & =-\frac{l^{(n-1)} \cos \alpha}{2 \pi^{2} r_{\gamma}} \operatorname{Im} \int d s_{1}^{\prime} d s_{2}^{\prime} \frac{k \exp \left\{i k l^{(n-1)}+\frac{i k}{2}\left(a_{2}+d_{2}\right) s_{1}^{\prime 2}-i \frac{\pi}{2} \nu^{(n-1)}\right\}}{\sqrt{\mid k^{2} \operatorname{det} B^{(n-1) \mid}}} \\
& =\frac{l_{\gamma} \rho \cos \alpha}{\pi r_{\gamma} N} \operatorname{Re}\left[\sqrt{\frac{2 \pi k}{\left|k \mathcal{M}_{42} \cos ^{2} \alpha+k \mathcal{M}_{31}\right|}} \exp \left\{i k l_{\gamma}-\frac{i \pi}{2} \mu_{\gamma}-\frac{i \pi}{4}\right\}\right],
\end{aligned}
$$

where $l_{\gamma}=l^{(n-1)}$ and

$$
\mu_{\gamma}=\nu^{(n-1)}+2+ \begin{cases}0 & \text { if }\left(a_{2}+d_{2}\right)>0 \\ 1 & \text { if }\left(a_{2}+d_{2}\right)<0\end{cases}
$$

In (38) the integral over $s_{2}^{\prime}$ was evaluated over the range $2 \pi \rho / N$ where $\rho$ is the distance of the stationary point on the billiard surface to the symmetry axis, and $N$ is the number of distinct rotations around the symmetry axis that map the orbit onto itself. The result (38) does not depend on the reflection point that is chosen as starting point of the trajectory, although this is not explicit from its form. The reason is again, that a semiclassical contribution to $\operatorname{Tr} \hat{Q}^{n}$ in (10) does not depend on the order in which the $n$ integrals are evaluated.

We acknowledge financial support by the Deutsche Forschungsgemeinschaft under contract No. DFG-Ste 241/6-1 and /7-2. 


\section{A General properties of the stability matrix}

The stability matrix $M$ is defined by the following relation

$$
\left(\begin{array}{l}
\mathrm{d} q_{2} \\
\mathrm{~d} q_{3} \\
\mathrm{~d} p_{2} \\
\mathrm{~d} p_{3}
\end{array}\right)=M\left(\begin{array}{l}
\mathrm{d} q_{2}^{\prime} \\
\mathrm{d} q_{3}^{\prime} \\
\mathrm{d} p_{2}^{\prime} \\
\mathrm{d} p_{3}^{\prime}
\end{array}\right), \quad M=\left(\begin{array}{llll}
M_{11} & M_{12} & M_{13} & M_{14} \\
M_{21} & M_{22} & M_{23} & M_{24} \\
M_{31} & M_{32} & M_{33} & M_{34} \\
M_{41} & M_{42} & M_{43} & M_{44}
\end{array}\right) .
$$

Here $\mathrm{d} q_{2}^{\prime}, \mathrm{d} q_{3}^{\prime}, \mathrm{d} p_{2}^{\prime}$, and $\mathrm{d} p_{3}^{\prime}$ are infinitesimal deviations from the considered trajectory at the starting point. They are given in a local coordinate system in which the first coordinate is along the trajectory, and the other two coordinates are perpendicular to it. The unprimed quantities $\mathrm{d} q_{2}, \mathrm{~d} q_{3}, \mathrm{~d} p_{2}$ and $\mathrm{d} p_{3}$ are the corresponding infinitesimal deviations at the end point of the trajectory. They follow from a linearization of the motion in the vicinity of the considered trajectory. In a compact notation

$$
\left(\begin{array}{l}
\mathrm{d} \vec{q}_{\perp} \\
\mathrm{d} \vec{p}_{\perp}
\end{array}\right)=M\left(\begin{array}{l}
\mathrm{d} \vec{q}_{\perp}^{\prime} \\
\mathrm{d} \vec{p}_{\perp}^{\prime}
\end{array}\right), \quad M=\left(\begin{array}{cc}
A & B \\
C & D
\end{array}\right)
$$

where $A, B, C$ and $D$ are $2 \times 2$-matrices.

The stability matrix is a symplectic matrix. It satisfies $M^{T} J M=J$, where $J$ denotes the matrix $J=\left(\begin{array}{cc}0 & I \\ -I & 0\end{array}\right)$ and $I$ is the two-dimensional unity matrix. The inverse of $M$ is given by $M^{-1}=-J M^{T} J$, and from $M^{-1} M=M M^{-1}=1$ one obtains the following relations between the submatrices of $M$

$$
\begin{array}{lll}
A^{T} C=C^{T} A, & B^{T} D=D^{T} B, & A^{T} D-C^{T} B=I, \\
A B^{T}=B A^{T}, & C D^{T}=D C^{T}, & A D^{T}-B C^{T}=I .
\end{array}
$$

Both rows of equations are equivalent to each other and to the symplectic condition for $M$. Altogether they yield 6 independent conditions for the matrix elements of $M$, so that only 10 elements of $M$ are independent.

Another useful quantity is the matrix $N$ which is defined in the following. It is obtained by solving the linear equations in (41) for $\vec{p}_{\perp}$ and $\vec{p}_{\perp}{ }^{\prime}$

$$
\left(\begin{array}{c}
\mathrm{d} \vec{p}_{\perp}^{\prime} \\
\mathrm{d} \vec{p}_{\perp}
\end{array}\right)=N\left(\begin{array}{c}
\mathrm{d} \vec{q}_{\perp}^{\prime} \\
\mathrm{d} \vec{q}_{\perp}
\end{array}\right)=\left(\begin{array}{cc}
\tilde{A} & \tilde{B} \\
\tilde{C} & \tilde{D}
\end{array}\right)\left(\begin{array}{c}
\mathrm{d} \vec{q}_{\perp}^{\prime} \\
\mathrm{d} \vec{q}_{\perp}
\end{array}\right)
$$

The relations between the submatrices of $N$ and those of $M$ are given by: $\tilde{A}=-B^{-1} A$, $\tilde{B}=B^{-1}, \tilde{C}=C-D B^{-1} A$, and $\tilde{D}=D B^{-1}$. On the other hand, the matrix elements of $N$ can be obtained from the action function of the trajectory $S\left(\vec{q}, \vec{q}^{\prime}, E\right)$ through

$$
N_{i j}=\sigma_{i} \frac{\partial^{2} S}{\partial z_{i} \partial z_{j}}, \quad \vec{z}=\left(\begin{array}{c}
\mathrm{d} \vec{q}_{\perp}^{\prime} \\
\mathrm{d} \vec{q}_{\perp}
\end{array}\right), \quad \sigma_{i}=\left\{\begin{array}{ll}
-1 & \text { if } j=1,2 \\
1 & \text { if } j=3,4
\end{array} .\right.
$$

Since the mixed second derivatives of the action function do not depend on the order of the derivation this yields 6 conditions for the matrix elements of $N$ which are equivalent to $\tilde{A}=\tilde{A}^{T}$, $\tilde{B}=-\tilde{C}^{T}$, and $\tilde{D}=\tilde{D}^{T}$. 


\section{B Determination of the stability matrix}

In two-dimensional billiard systems the stability matrix is composed of two kinds of partial stability matrices $M_{t}$ and $M_{r}$ where $M_{t}$ denotes the matrix for the free motion between two reflections and $M_{r}$ is the matrix for a reflection. They have the following form (see [35])

$$
M_{t}=\left(\begin{array}{cc}
1 & L / p \\
0 & 1
\end{array}\right), \quad M_{r}=\left(\begin{array}{cc}
-1 & 0 \\
\frac{2 p}{R \cos \alpha} & -1
\end{array}\right)
$$

where $L$ is the length of a trajectory between two reflections, $R$ is the radius of curvature at a reflection point, $\alpha$ is the angle of incidence, and $p$ is the modulus of the momentum.

In three dimensions there is an additional kind of matrix which describes a rotation of the local coordinate system around the trajectory [12]. The stability matrix for a trajectory from a point $\vec{r}_{a}$ to a point $\vec{r}_{b}$ with $n$ reflections on the boundary can be written in the following form

$$
M=M_{T}^{b \leftarrow n} M_{R}^{n} M_{S}^{n} \ldots M_{T}^{3 \leftarrow 2} M_{R}^{2} M_{S}^{2} M_{T}^{2 \leftarrow 1} M_{R}^{1} M_{S}^{1} M_{T}^{1 \leftarrow a} .
$$

Here $M_{T}$ is the matrix for a part of the trajectory of length $L$ between two reflections. It is a straightforward generalization of the matrix $M_{t}$ in two dimensions. $M_{S}$ corresponds to a rotation of the local coordinate system around the trajectory such that the new coordinate with index 2 lies in the reflection plane that is spanned by the incoming and outgoing trajectory at a reflection point. The angle of rotation $\theta$ is defined by

$$
\begin{array}{ll}
\hat{e}_{2}=\hat{e}_{2}^{\prime} \cos \theta+\hat{e}_{3}^{\prime} \sin \theta, & \mathrm{d} q_{2}=\mathrm{d} q_{2}^{\prime} \cos \theta+\mathrm{d} q_{3}^{\prime} \sin \theta, \\
\hat{e}_{3}=-\hat{e}_{2}^{\prime} \sin \theta+\hat{e}_{3}^{\prime} \cos \theta, & \mathrm{d} q_{3}=-\mathrm{d} q_{2}^{\prime} \sin \theta+\mathrm{d} q_{3}^{\prime} \cos \theta,
\end{array}
$$

where $\hat{e}_{i}^{\prime}$ are the unit vectors along the old coordinate axes and $\hat{e}_{i}$ are the unit vectors along the new coordinate axes. $M_{T}$ and $M_{S}$ are given by

$$
M_{T}=\left(\begin{array}{cccc}
1 & 0 & L / p & 0 \\
0 & 1 & 0 & L / p \\
0 & 0 & 1 & 0 \\
0 & 0 & 0 & 1
\end{array}\right), \quad M_{S}=\left(\begin{array}{cccc}
\cos \theta & \sin \theta & 0 & 0 \\
-\sin \theta & \cos \theta & 0 & 0 \\
0 & 0 & \cos \theta & \sin \theta \\
0 & 0 & -\sin \theta & \cos \theta
\end{array}\right) .
$$

Furthermore, $M_{R}$ is the matrix for a reflection

$$
M_{R}=\left(\begin{array}{cccc}
-1 & 0 & 0 & 0 \\
0 & 1 & 0 & 0 \\
\frac{2 p}{R_{a} \cos \alpha} & \frac{2 p}{R_{c}} & -1 & 0 \\
-\frac{2 p}{R_{c}} & -\frac{2 p \cos \alpha}{R_{b}} & 0 & 1
\end{array}\right) \text {. }
$$

Here $\alpha$ is the angle of incidence, i. e. the angle between the incoming (or outgoing) trajectory and the normal to the boundary. $R_{a}$ and $R_{b}$ are the radii of curvature in the reflection plane and perpendicular to it, respectively. $R_{a}, R_{b}$ and $R_{c}$ can be expressed in terms of the two main radii of curvature at the reflection point $R_{1}$ and $R_{2}$, and the angle $\beta$ between the tangent lying in the reflection plane and the direction of the main curvature $1 / R_{1}$ (see eq. (57)).

$$
\frac{1}{R_{a}}=\frac{\cos ^{2} \beta}{R_{1}}+\frac{\sin ^{2} \beta}{R_{2}}, \quad \frac{1}{R_{b}}=\frac{\sin ^{2} \beta}{R_{1}}+\frac{\cos ^{2} \beta}{R_{2}}, \quad \frac{1}{R_{c}}=\frac{R_{2}-R_{1}}{R_{1} R_{2}} \cos \beta \sin \beta .
$$


The form of the matrices $M_{T}, M_{S}$ and $M_{R}$ has been given in [12] for the case of reflections on spheres $\left(R_{1}=R_{2}\right)$. In 36] these results were generalized to cases where one of the main curvature directions of the billiard boundary lies in the reflection plane $(\theta=0)$. Here we give the form of $M_{R}$ for arbitrary reflections. The derivation is carried out in appendix $\mathrm{D}$.

Finally, we discuss the stability matrix for a periodic orbit. It is given by (46) if $a$ is replaced by $n$ and the term $M_{T}^{b \leftarrow n}$ is dropped (see eq. (51)). In this form it is assumed that the local coordinate system after one traversal of the trajectory has the same orientation as it has at the beginning. Otherwise an additional rotation matrix $M_{S}$ has to be multiplied to the product. However, since one has the freedom to choose the orientation of the local coordinate system at the starting point, one can always choose it to have the same orientation as at the end point. In other words, for the evaluation of (51) from right to left one starts with a local coordinate systems whose second coordinate lies in the reflection plane at $\vec{r}_{n}$.

\section{Determination of the Maslov index}

We summarize in this appendix the results of sections 3 and 4 for the Maslov index of a trajectory. Consider a trajectory with $n$ reflections on the billiard wall. If one of the reflection points is chosen as starting point of the trajectory the stability matrix can be written in the following form

$$
M=M_{R}^{n} M_{S}^{n} M_{T}^{n \leftarrow n-1} \ldots M_{T}^{3 \leftarrow 2} M_{R}^{2} M_{S}^{2} M_{T}^{2 \leftarrow 1} M_{R}^{1} M_{S}^{1} M_{T}^{1 \leftarrow n}
$$

Here the orientation of the local coordinate system at the initial point has to agree with its orientation at the final point as discussed in the appendix B. Now the trajectory is traversed once, and correspondingly the multiplication of partial matrices in (51) is carried out. The Maslov index changes by two for every reflection matrix in this product (in case of Dirichlet boundary conditions). Otherwise it changes only during a part of the trajectory between two

reflections. How much it changes is determined by the following method. For every $M_{T}$-matrix in (51) except for the first one $M_{T}^{1 \leftarrow n}$ one denotes the product of partial matrices up to this $M_{T}$-matrix by $M^{\prime}$, and the product including this $M_{T}$-matrix by $M$ such that $M=M_{T} M^{\prime}$. Then one considers the following two quantities

$$
Z_{1}=\frac{M_{13} M_{24}^{\prime}-M_{14} M_{23}^{\prime}}{M_{13}^{\prime} M_{24}^{\prime}-M_{14}^{\prime} M_{23}^{\prime}}, \quad Z_{2}=\frac{M_{13} M_{24}-M_{14} M_{23}}{M_{13}^{\prime} M_{24}^{\prime}-M_{14}^{\prime} M_{23}^{\prime}} .
$$

The Maslov index changes according to the following rule

$$
\nu=\nu^{\prime}+ \begin{cases}0 & \text { if } Z_{1}>0 \text { and } Z_{2}>0 \\ 1 & \text { if } Z_{2}<0 \\ 2 & \text { if } Z_{1}<0 \text { and } Z_{2}>0\end{cases}
$$

where $\nu^{\prime}$ denotes the previous value of the index. This follows from (25) and (28) by noting that the $M_{1 i}$-element change only their sign upon a reflection and the $M_{2 i}$-elements remain unchanged. (Note that $M^{\prime}$ is defined here including the reflection matrix in contrast to the main section.)

Finally there can be an additional change of the Maslov index that arises from the evaluation of the trace. It is determined by considering

$$
Z_{1}=\frac{\mathcal{M}_{42}}{\operatorname{det} B}, \quad Z_{2}=\frac{\operatorname{det}(M-1)}{\operatorname{det} B},
$$


where $M$ is now the total stability matrix of the periodic orbit given in (51), and $B$ is its upper right submatrix as defined in appendix A. $\mathcal{M}_{i j}$ is the determinant of the $3 \times 3$-matrix that is obtained by dropping the $i$-th row and the $j$-th column of the matrix $(M-1)$.

Then the index $\nu$ is changed according to

$$
\mu=\nu+ \begin{cases}0 & \text { if } Z_{1}>0 \text { and } Z_{2}>0 \\ 1 & \text { if } Z_{2}<0 \\ 2 & \text { if } Z_{1}<0 \text { and } Z_{2}>0\end{cases}
$$

This yields the total Maslov index $\mu$ of the periodic orbit.

\section{Derivation of the stability matrix for a reflection}

Let the point of reflection be at the origin of the coordinate system. The $x_{1}^{\prime}$ - and $x_{2}^{\prime}$-axes of the coordinate system are chosen to lie in the directions of the two main curvatures of the boundary surface. Then the boundary is locally represented by

$$
x_{3}^{\prime}=-\frac{x_{1}^{\prime 2}}{2 R_{1}}-\frac{x_{2}^{\prime 2}}{2 R_{2}}
$$

where $R_{1}$ and $R_{2}$ are the two main radii of curvature. Now the coordinate system is rotated about the $x_{3}^{\prime}$-axis so that the new $x_{1}$-axis lies in the plane of reflection, and the component of the incoming momentum in $x_{1}$-direction is non-negative

$$
x_{1}^{\prime}=x_{1} \cos \beta+x_{2} \sin \beta, \quad x_{2}^{\prime}=-x_{1} \sin \beta+x_{2} \cos \beta, \quad x_{3}^{\prime}=x_{3} .
$$

In the new coordinates the boundary is locally represented by

$$
x_{3}=-\frac{x_{1}^{2}}{2 R_{a}}-\frac{x_{2}^{2}}{2 R_{b}}-\frac{x_{1} x_{2}}{R_{c}}
$$

where $R_{a}$ and $R_{b}$ are the radii of curvature in direction of the new coordinate axes in planes containing the normal vector, and the relations between $R_{a}, R_{b}, R_{c}$ and $R_{1}, R_{2}$ and $\beta$ are given in (50).

Quantities before the reflection are given a prime in the following, and the conserved absolute value of the momentum is denoted by $p$. The angle of incidence $\alpha$ is defined as the angle between the incoming momentum $\vec{p}^{\prime}$ and the outward pointing normal vector to the boundary $\hat{n}, 0 \leq \alpha<\pi / 2$. The outgoing momentum is $\vec{p}$. One has

$$
\hat{n}=\left(\begin{array}{l}
0 \\
0 \\
1
\end{array}\right), \quad \vec{p}^{\prime}=p\left(\begin{array}{c}
\sin \alpha \\
0 \\
\cos \alpha
\end{array}\right), \quad \vec{p}=\vec{p}^{\prime}-2\left(\vec{p}^{\prime} \cdot \hat{n}\right) \hat{n}=p\left(\begin{array}{c}
\sin \alpha \\
0 \\
-\cos \alpha
\end{array}\right) .
$$

In the following one considers an infinitesimal change in the position and the momentum of the incoming trajectory and determines the corresponding deviations for the outgoing trajectory. First we discuss how the normal vector changes if the point of reflection is changed. The new 
normal vector is determined from two tangential vectors at the point $\vec{r}=\left(x_{1}, x_{2}, x_{3}\right)$ with $x_{3}$ given by eq. (58)

$$
\overrightarrow{t_{1}}=\frac{\partial \vec{r}}{\partial x_{1}}=\left(\begin{array}{c}
1 \\
0 \\
-\frac{x_{1}}{R_{a}}-\frac{x_{2}}{R_{c}}
\end{array}\right), \quad \overrightarrow{t_{2}}=\frac{\partial \vec{r}}{\partial x_{2}}=\left(\begin{array}{c}
0 \\
1 \\
-\frac{x_{2}}{R_{b}}-\frac{x_{1}}{R_{c}}
\end{array}\right)
$$

from which one obtains

$$
\hat{n}=\frac{\overrightarrow{t_{1}} \times \overrightarrow{t_{2}}}{\left|\overrightarrow{t_{1}} \times \overrightarrow{t_{2}}\right|}=\left(\begin{array}{c}
\frac{x_{1}}{R_{a}}+\frac{x_{2}}{R_{c}} \\
\frac{x_{2}}{R_{b}}+\frac{x_{1}}{R_{c}} \\
1
\end{array}\right)\left[1+\left(\frac{x_{1}}{R_{a}}+\frac{x_{2}}{R_{c}}\right)^{2}+\left(\frac{x_{2}}{R_{b}}+\frac{x_{1}}{R_{c}}\right)^{2}\right]^{-1 / 2} .
$$

Now one considers the four cases that one of the infinitesimal quantities $\mathrm{d} q_{2}^{\prime}, \mathrm{d} q_{3}^{\prime}, \mathrm{d} p_{2}^{\prime}$ and $\mathrm{d} p_{3}^{\prime}$ is different from zero. They describe infinitesimal deviations from the incoming trajectory in position and momentum, respectively, immediately before the reflection. They are expressed in a local coordinate system, where the first coordinate is parallel to the trajectory and the other two coordinates are perpendicular to it. The quantities with index 2 are in the plane of reflection and those with index 3 perpendicular to it. For each of these four cases the corresponding deviations for the outgoing trajectory immediately after the reflection are determined. They are written without a prime.

$\mathrm{d} p_{2}^{\prime} \neq 0$ : One sees easily that only $\mathrm{d} p_{2}$ is different from zero: $\mathrm{d} q_{2}=0, \mathrm{~d} q_{3}=0, \mathrm{~d} p_{2}=-\mathrm{d} p_{2}^{\prime}$ and $\mathrm{d} p_{3}=0$.

$\underline{\mathrm{d} p_{3}^{\prime} \neq 0}$ : Now only $\mathrm{d} p_{3}$ is different from zero: $\mathrm{d} q_{2}=0, \mathrm{~d} q_{3}=0, \mathrm{~d} p_{2}=0$ and $\mathrm{d} p_{3}=\mathrm{d} p_{3}^{\prime}$.

$\mathrm{d} q_{2}^{\prime} \neq 0$ : The deviation of the point of reflection is given by $\mathrm{d} x_{1}=\mathrm{d} q_{2}^{\prime} / \cos \alpha=-\mathrm{d} q_{2} / \cos \alpha$ and $\mathrm{d} x_{2}=0$. It follows that $\mathrm{d} q_{2}=-\mathrm{d} q_{2}^{\prime}$ and $\mathrm{d} q_{3}=0$. With the normal vector in (61), and with $\vec{p}^{\prime}$ and the relation for $\vec{p}$ in (59) one finds:

$$
\vec{n}=\left(\begin{array}{c}
\frac{\mathrm{d} q_{2}^{\prime}}{R_{a} \cos \alpha} \\
\frac{\mathrm{d} q_{2}^{\prime}}{R_{c} \cos \alpha} \\
1
\end{array}\right), \quad \vec{p}=p\left(\begin{array}{c}
\sin \alpha-\frac{2 \mathrm{~d} q_{2}^{\prime}}{R_{a}} \\
-\frac{2 \mathrm{~d} q_{2}^{\prime}}{R_{c}} \\
-\cos \alpha-\frac{2 \tan \alpha \mathrm{d} q_{2}^{\prime}}{R_{a}}
\end{array}\right)=\left(\begin{array}{c}
p \sin \alpha-\cos \alpha \mathrm{d} p_{2} \\
\mathrm{~d} p_{3} \\
-p \cos \alpha-\sin \alpha \mathrm{d} p_{2}
\end{array}\right) .
$$

From this follows that $\mathrm{d} p_{2}=2 p \mathrm{~d} q_{2}^{\prime} /\left(R_{a} \cos \alpha\right)$ and $\mathrm{d} p_{3}=-2 p \mathrm{~d} q_{2}^{\prime} / R_{c}$.

$\mathrm{d} q_{3}^{\prime} \neq 0$ : The deviation of the point of reflection is given by $\mathrm{d} x_{1}=0$ and $\mathrm{d} x_{2}=\mathrm{d} q_{3}^{\prime}=\mathrm{d} q_{3}$, and it follows that $\mathrm{d} q_{2}=0$ and $\mathrm{d} q_{3}=\mathrm{d} q_{3}^{\prime}$. With the normal vector in (61), and with $\vec{p}^{\prime}$ and 
the relation for $\vec{p}$ in (59) one finds:

$$
\vec{n}=\left(\begin{array}{c}
\frac{\mathrm{d} q_{3}^{\prime}}{R_{c}} \\
\frac{\mathrm{d} q_{3}^{\prime}}{R_{b}} \\
1
\end{array}\right), \quad \vec{p}=p\left(\begin{array}{c}
\sin \alpha-\frac{2 \cos \alpha \mathrm{d} q_{3}^{\prime}}{R_{c}} \\
-\frac{2 \cos \alpha \mathrm{d} q_{3}^{\prime}}{R_{b}} \\
-\cos \alpha-\frac{2 \sin \alpha \mathrm{d} q_{3}^{\prime}}{R_{c}}
\end{array}\right)=\left(\begin{array}{c}
p \sin \alpha-\cos \alpha \mathrm{d} p_{2} \\
\mathrm{~d} p_{3} \\
-p \cos \alpha-\sin \alpha \mathrm{d} p_{2}
\end{array}\right) .
$$

From this follows that $\mathrm{d} p_{2}=2 p \mathrm{~d} q_{3}^{\prime} / R_{c}$ and $\mathrm{d} p_{3}=-2 p \cos \alpha \mathrm{d} q_{3}^{\prime} / R_{b}$.

Collecting all results one obtains the form of the stability matrix for a reflection in (49).

\section{References}

[1] H. Primack and U. Smilansky: Quantization of the Three-Dimensional Sinai Billiard, Phys. Rev. Lett. 74 (1995) 4831-4834.

[2] C. Matthies: Picards Billard. Ein Modell für Arithmetisches Quantenchaos in drei Dimensionen, Ph.D. thesis, Universität Hamburg, (1995).

[3] W. Huntebrinker: Numerische Bestimmung von Eigenwerten des Laplace-Beltrami-Operators auf dreidimensionalen hyperbolischen Räumen mit Finite-Element-Methoden, Ph.D. thesis, Universität Düsseldorf, (1995).

[4] R. Aurich and J. Marklof: Trace Formulae for Three-Dimensional Hyperbolic Lattices and Application to a Strongly Chaotic Tetrahedral Billiard, Physica D 92 (1996) 101-129.

[5] H. Alt, H.-D. Gräf, R. Hofferbert, C. Rangacharyulu, H. Rehfeld, A. Richter, P. Schardt and A. Wirzba: Chaotic Dynamics in a Three-Dimensional Superconducting Microwave Billiard, Phys. Rev. E 54 (1996) 2303-2312.

[6] T. Prosen: Quantization of Generic Chaotic 3D Billiard with Smooth Boundary I: Energy Level Statistics, (1996), preprint.

[7] T. Prosen: Quantization of Generic Chaotic 3D Billiard with Smooth Boundary II: Structure of High-Lying Eigenstates, (1996), preprint.

[8] G. Steil: Eigenvalues of the Laplacian for Bianchi groups, (1997), preprint.

[9] L. A. Bunimovich and J. Rehacek: Nowhere Dispersing 3D Billiards with Non-vanishing Lyapunov Exponents, (1997), preprint.

[10] L. A. Bunimovich and J. Rehacek: How High-Dimensional Stadia Look Like, (1997), preprint.

[11] M. Henseler, A. Wirzba and T. Guhr: Quantization of Hyperbolic N-Sphere Scattering Systems in Three Dimensions, Ann. Phys. 258 (1997) 286-319.

[12] H. Primack: Quantal and Semiclassical Analysis of the Three-Dimensional Sinai Billiard, Ph.D. thesis, Weizmann Institute of Science, (1997). 
[13] M. Brack and R. K. Bhaduri: Semiclassical Physics, Addison and Wesley, Reading, (1997).

[14] R. Balian and C. Bloch: Distribution of Eigenfrequencies for the Wave Equation in a Finite Domain: I. Three-Dimensional Problem with Smooth Boundary Surface, Ann. Phys. 60 (1970) 401-447, Erratum in Ann. Phys. 84 (1974) 559.

[15] R. Balian and C. Bloch: Distribution of Eigenfrequencies for the Wave Equation in a Finite Domain: II. Electromagnetic Field. Riemannian Spaces, Ann. Phys. 64 (1971) 271-307, Erratum in Ann. Phys. 84 (1974) 559-562.

[16] R. Balian and C. Bloch: Distribution of Eigenfrequencies for the Wave Equation in a Finite Domain: III. Eigenfrequency Density Oscillations, Ann. Phys. 69 (1972) 76-160.

[17] M. C. Gutzwiller: Chaos in Classical and Quantum Mechanics, Springer, New York, (1990).

[18] T. Harayama and A. Shudo: Zeta Function Derived from the Boundary Element Method, J. Phys. A 165 (1992) 417-426.

[19] B. Burmeister: Korrekturen zur Gutzwillerschen Spurformel für Quantenbillards, (1995), Diploma thesis, Universität Hamburg.

[20] M. Sieber, N. Pavloff and C. Schmit: Uniform Approximation for Diffractive Contributions to the Trace Formula in Billiard Systems, Phys. Rev. E 55 (1997) 2279-2299.

[21] M. Brack, S. M. Reimann and M. Sieber: Semiclassical Interpretation of the Mass Asymmetry in Nuclear Fission, Phys. Rev. Lett. 79 (1997) 1817-1820.

[22] R. E. Kleinman and G. F. Roach: Boundary Integral Equations for the Three-Dimensional Helmholtz Equation, SIAM Review 16 (1974) 214-236.

[23] J. R. J. Riddell: Boundary-Distribution Solution of the Helmholtz Equation for a Region with Corners, J. Comp. Phys. 31 (1979) 21-41.

[24] J. R. J. Riddell: Numerical Solution of the Helmholtz Equation for Two-Dimensional Polygonal Regions, J. Comp. Phys. 31 (1979) 42-59.

[25] B. Georgeot and R. E. Prange: Exact and Quasiclassical Fredholm Solutions of Quantum Billiards, Phys. Rev. Lett. 74 (1995) 2851-2854.

[26] T. Harayama, A. Shudo and S. Tasaki: Resummation of the Gutzwiller-Voros Zeta Function for Semiclassical Quantization and Resonance Scattering in Strongly Chaotic Systems, (1997), preprint.

[27] B. Burmeister and F. Steiner: (1995), unpublished.

[28] S. Tasaki, T. Harayama and A. Shudo: Interior Dirichlet Eigenvalue Problem, Exterior Neumann Scattering Problem, and the Boundary Element Method for Quantum Billiards, Phys. Rev. E 56 (1997) R13-R16.

[29] J.-P. Eckmann and C.-A. Pillet: Zeta Functions with Dirichlet and Neumann Boundary Conditions for Exterior Domains, Helvetica Physica Acta 70 (1997) 44-65. 
[30] U. Smilansky and I. Ussishkin: The Smooth Spectral Counting Function and the Total Phase Shift for Quantum Billiards, J. Phys. A 29 (1996) 2587-2597.

[31] T. Hesse: Semiklassische Untersuchung zwei- und dreidimensionaler Billardsysteme, Ph.D. thesis, Universität Ulm, (1997).

[32] S. Fenyö and H. W. Stolle: Theorie und Praxis der linearen Integralgleichungen 2, Birkhäuser, Basel, (1983).

[33] S. C. Creagh, J. M. Robbins and R. G. Littlejohn: Geometrical Properties of Maslov Indices in the Semiclassical Trace Formula for the Density of States, Phys. Rev. A 42 (1990) 1907-1922.

[34] S. C. Creagh and R. G. Littlejohn: Semiclassical Trace Formulas in the Presence of Continuous Symmetries, Phys. Rev. A 44 (1991) 836-850.

[35] M. Sieber and F. Steiner: Classical and quantum mechanics of a strongly chaotic billiard system, Physica D 44 (1990) 248-266.

[36] A. Wirzba: Non-paper about 3-d Jacobians, (1995), unpublished. 